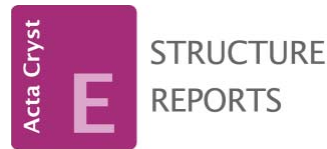

OPEN $\curvearrowright$ ACCESS

ISSN 1600-5368

\section{Crystal structure of (3E)-3-(2,4-dinitro- phenoxymethyl)-4-phenylbut-3-en-2-one}

Ignez Caracelli, ${ }^{\text {a* }}$ Stella H. Maganhi, ${ }^{a}$ Paulo J. S. Moran, ${ }^{\text {b }}$ Bruno R. S. de Paula, ${ }^{b}$ Felix N. Delling ${ }^{c}$ and Edward R. T. Tiekink $^{\mathrm{d}}$

aBioMat-Departmento de Física, Universidade Federal de São Carlos, 13565-905 São Carlos, SP, Brazil, ' Instituto de Química, Universidade Estadual de Campinas, CP 6154, 13083-970 Campinas, SP, Brazil, ' Departmento de Qímica, Universidade Federal de São Carlos, 13565-905 São Carlos, SP, Brazil, and ${ }^{\mathbf{d}}$ Department of Chemistry, University of Malaya, 50603 Kuala Lumpur, Malaysia. *Correspondence e-mail: ignez@ufscar.br

Received 15 August 2014; accepted 19 August 2014

Edited by P. C. Healy, Griffith University, Australia

In the title compound, $\mathrm{C}_{17} \mathrm{H}_{14} \mathrm{~N}_{2} \mathrm{O}_{6}$, the conformation about the $\mathrm{C}=\mathrm{C}$ double bond $[1.345(2) \AA]$ is $E$, with the ketone moiety almost coplanar $\left[\mathrm{C}-\mathrm{C}-\mathrm{C}-\mathrm{C}\right.$ torsion angle $\left.=9.5(2)^{\circ}\right]$ along with the phenyl ring $\left[\mathrm{C}-\mathrm{C}-\mathrm{C}-\mathrm{C}=5.9(2)^{\circ}\right]$. The aromatic rings are almost perpendicular to each other [dihedral angle $=86.66(7)^{\circ}$ ]. The 4-nitro moiety is approximately coplanar with the benzene ring to which it is attached $\left[\mathrm{O}-\mathrm{N}-\mathrm{C}-\mathrm{C}=4.2(2)^{\circ}\right]$, whereas the one in the ortho position is twisted $\left[\mathrm{O}-\mathrm{N}-\mathrm{C}-\mathrm{C}=138.28(13)^{\circ}\right]$. The molecules associate via $\mathrm{C}-\mathrm{H} \cdots \mathrm{O}$ interactions, involving both $\mathrm{O}$ atoms from the 2-nitro group, to form a helical supramolecular chain along [010]. Nitro-nitro $\mathrm{N} \cdots \mathrm{O}$ interactions [2.8461 (19) $\AA$ A ] connect the chains into layers that stack along [001].

Keywords: crystal structure; hydrogen bonding; $\mathrm{N} \cdots \mathrm{O}$ interactions.

CCDC reference: 1020026

\section{Related literature}

For background to biotransformations mediated by Saccharomyces cerevisiae, see: Rodrigues et al. (2004); de Paula et al. (2013). For a related structure, see: Zukerman-Schpector et al. (2014). For interactions between nitro groups, see: Daszkiewicz (2013).

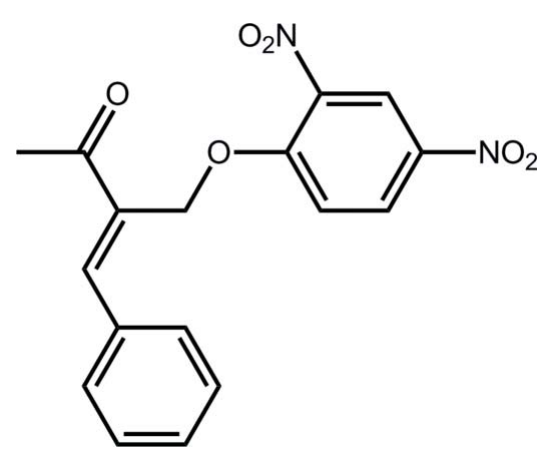

\section{Experimental}

\subsection{Crystal data}

$\mathrm{C}_{17} \mathrm{H}_{14} \mathrm{~N}_{2} \mathrm{O}_{6}$

Monoclinic, $P 2_{1} / c$

$a=12.8459(6) \AA$

$b=7.6983(4) \AA$

$c=19.4283(8) \AA$

$\beta=122.254(2)^{\circ}$

$$
\begin{aligned}
& V=1624.82(14) \AA^{3} \\
& Z=4 \\
& \text { Mo } K \alpha \text { radiation } \\
& \mu=0.11 \mathrm{~mm}^{-1} \\
& T=290 \mathrm{~K} \\
& 0.66 \times 0.45 \times 0.28 \mathrm{~mm}
\end{aligned}
$$

\subsection{Data collection}

Bruker Kappa APEXII CCD diffractometer

Absorption correction: multi-scan (SADABS; Sheldrick, 1996)

$T_{\min }=0.699, T_{\max }=0.745$

10435 measured reflections 2957 independent reflections 2630 reflections with $I>2 \sigma(I)$ $R_{\text {int }}=0.019$

\subsection{Refinement}

$R\left[F^{2}>2 \sigma\left(F^{2}\right)\right]=0.037$

$w R\left(F^{2}\right)=0.101$

$S=1.04$

2957 reflections

228 parameters

$\mathrm{H}$-atom parameters constrained

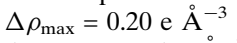

$\Delta \rho_{\min }=-0.21$ e $\AA^{-3}$

Table 1

Hydrogen-bond geometry $\left(\AA{ }^{\circ}\right)$.

\begin{tabular}{lllll}
\hline$D-\mathrm{H} \cdots A$ & $D-\mathrm{H}$ & $\mathrm{H} \cdots A$ & $D \cdots A$ & $D-\mathrm{H} \cdots A$ \\
\hline $\mathrm{C} 6-\mathrm{H} 6 \cdots \mathrm{O}^{\mathrm{i}}$ & 0.93 & 2.41 & $3.1375(18)$ & 135 \\
$\mathrm{C} 16-\mathrm{H} 16 \cdots \mathrm{O}^{\mathrm{ii}}$ & 0.93 & 2.58 & $3.292(3)$ & 134 \\
\hline
\end{tabular}

Symmetry codes: (i) $x, y+1, z$; (ii) $-x+1, y+\frac{1}{2},-z+\frac{1}{2}$.

Data collection: APEX2 (Bruker, 2009); cell refinement: SAINT (Bruker, 2009); data reduction: SAINT; program(s) used to solve structure: SIR97 (Altomare et al., 1999); program(s) used to refine structure: SHELXL97 (Sheldrick, 2008); molecular graphics: ORTEP-3 for Windows (Farrugia, 2012), QMol (Gans \& Shalloway, 2001) and DIAMOND (Brandenburg, 2006); software used to prepare material for publication: MarvinSketch (ChemAxon, 2010) and publCIF (Westrip, 2010).

\section{Acknowledgements}

We thank Professor Regina H. A. Santos from IQSC-USP for the data collection. The Brazilian agencies CNPq (306121/ 2013-1 to IC), CAPES (808/2009 to IC) and FAPESP (2012/ 22524-9 to SHM) are acknowledged for financial support. 


\section{data reports}

Supporting information for this paper is available from the $\mathrm{IUCr}$ electronic archives (Reference: HG5406).

\section{References}

Altomare, A., Burla, M. C., Camalli, M., Cascarano, G. L., Giacovazzo, C., Guagliardi, A., Moliterni, A. G. G., Polidori, G. \& Spagna, R. (1999). J. Appl. Cryst. 32, 115-119.

Brandenburg, K. (2006). DIAMOND. Crystal Impact GbR, Bonn, Germany. Bruker (2009). APEX2 and SAINT. Bruker AXS Inc., Madison, Wisconsin, USA.
ChemAxon (2010). Marvinsketch. http://www.chemaxon.com

Daszkiewicz, M. (2013). CrystEngComm, 15, 10427-10430.

Farrugia, L. J. (2012). J. Appl. Cryst. 45, 849-854.

Gans, J. \& Shalloway, D. (2001). J. Mol. Graph. Model. 19, 557-559.

Paula, B. R. S. de, Zampieri, D. S., Rodrigues, J. A. R. \& Moran, P. J. S. (2013). Tetrahedron Asymmetry, 24, 973-981.

Rodrigues, J. A. R., Moran, P. J. S., Conceicão, G. J. A. \& Fardelone, L. C. (2004). Food Technol. Biotechnol. 42, 295-303.

Sheldrick, G. M. (1996). SADABS. University of Göttingen, Germany.

Sheldrick, G. M. (2008). Acta Cryst. A64, 112-122.

Westrip, S. P. (2010). J. Appl. Cryst. 43, 920-925.

Zukerman-Schpector, J., Maganhi, S. H., Moran, P. J. S., de Paula, B. R. S., Nucci, P. R. \& Tiekink, E. R. T. (2014). Acta Cryst. E70, o1020-o1021. 


\section{supporting information}

Acta Cryst. (2014). E70, o1051-o1052［doi:10.1107/S1600536814018819]

\section{Crystal structure of (3E)-3-(2,4-dinitrophenoxymethyl)-4-phenylbut-3-en-2-one}

Ignez Caracelli, Stella H. Maganhi, Paulo J. S. Moran, Bruno R. S. de Paula, Felix N. Delling and Edward R. T. Tiekink

\section{S1. Structural commentary}

In the context of the study of biotransformation reactions mediated by Saccharomyces cerevisiae, such as the bioreduction of $\alpha$-haloketones and enones (Rodrigues et al., 2004), the title compound, (3E)-3-(2,4-dinitrophenoxymethyl)-4-phenylbut-3-en-2- one, (I), as well as its 4-nitrophenylmethyl analogue, i.e. (3E)-3-(4-nitrophenoxymethyl)-4phenylbut-3-en-2-one, (II), (Zukerman-Schpector et al. 2014), were synthesised to be used as substrates in order to compare their behaviour with that of the 3-halomethyl-4-phenyl-3-buten-2-ones analogues (de Paula et al., 2013). Herein, the crystal structure determination and spectroscopic details of (I) are described.

In (I), the conformation about the $\mathrm{C}=\mathrm{C}$ double bond $[1.345$ (2) $\AA$ ] is $E$. The ketone moiety almost co-planar [C11-C8$\mathrm{C} 9-\mathrm{C} 10$ torsion angle $\left.=9.5(2)^{\circ}\right]$ with the double bond but the phenyl ring is twisted $[\mathrm{C} 8-\mathrm{C} 11-\mathrm{C} 12-\mathrm{C} 17=$ $-150.23(16)^{\circ}$ ]. The aromatic rings are almost perpendicular to each other [dihedral angle $=86.66(7)^{\circ}$ ]. The $p$-nitro moiety is approximately co-planar with the benzene ring to which it is attached [O3-N1-C4-C5 $=-175.29$ (14) ${ }^{\circ}$ ] while the the one in the $o$-position is twisted [O5-N2-C2-C3 $\left.=-40.76(17)^{\circ}\right]$.

Fig. 2 shows and overlay diagram of (I) and the inverted molecule of (II) (Zukerman-Schpector et al. 2014). Clearly, the two molecules adopt very similar conformations.

In the crystal packing, molecules associate via $\mathrm{C}-\mathrm{H} \cdots \mathrm{O}$ interactions, whereby the $\mathrm{O}$ atoms of the $o$-nitro group interact with $\mathrm{H}$ derived from a benzene or a phenyl ring, leading to a supramolecular helical chain along [0 10$]$; Fig. 2. There also

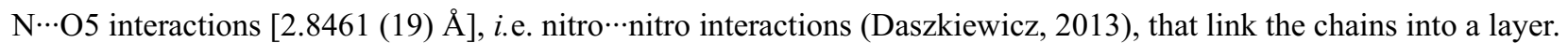
Layers stack along [ $\left[\begin{array}{lll}0 & 0 & 1\end{array}\right]$ without specific intermolecular interactions between them, Fig. 3.

\section{S2. Synthesis and crystallization}

Potassium carbonate (232 mg, $2.4 \mathrm{mmol}$ ) and 2,4-dinitrophenol (368 mg, $2 \mathrm{mmol}$ ) were added to a solution of 4-nitrophenol (1.53 g, $11 \mathrm{mmol})$ and 3-bromomethyl-4-phenyl-3-buten-2-one (478 $\mathrm{mg}, 2 \mathrm{mmol})$ in dry acetone $(4 \mathrm{~mL})$. The reaction mixture was stirred for 16 hours and the filtered. The solvent was evaporated and the residue was purified by column chromatography (hexane/EtOAc, 80:20) to afford the product as a colourless solid in $75 \%$ yield. The product was recrystallized by slow evaporation of a 2:1 dichloromethane/hexane mixture. $M . p t: 442.2-442.8 \mathrm{~K} .{ }^{1} \mathrm{H} \mathrm{NMR}\left(\mathrm{CD}_{2} \mathrm{Cl}_{2}\right.$, $400 \mathrm{MHz}): \delta 2.52(3 \mathrm{H}, \mathrm{s}), 5.12(2 \mathrm{H}, \mathrm{s}), 7.41(\mathrm{~d}, 1 H, J=9.2 \mathrm{~Hz}), 7.43-7.56(5 H, \mathrm{~m}), 7.99(1 H, \mathrm{~s}), 8.42(\mathrm{dd}, 1 H, J=2.8$, $9.2 \mathrm{~Hz}), 8.72(\mathrm{~d}, 1 H, J=2.8 \mathrm{~Hz}) \cdot{ }^{13} \mathrm{C} \mathrm{NMR}\left(\mathrm{CD}_{2} \mathrm{Cl}_{2}, 150 \mathrm{MHz}\right): \delta 26.2,64.7,116.1,122.3,129.5,129.7,130.2,130.8$, $134.5,134.6,139.8,140.9,148.1,157.0,198.4$.

\section{S3. Refinement}

Carbon-bound H-atoms were placed in calculated positions ( $\mathrm{C}-\mathrm{H} 0.93$ to $0.97 \AA$ ) and were included in the refinement in the riding model approximation, with $U_{i s o}(\mathrm{H})=1.2 U_{e q}(\mathrm{C})$ and $1.5 U_{e q}($ methyl-C). 


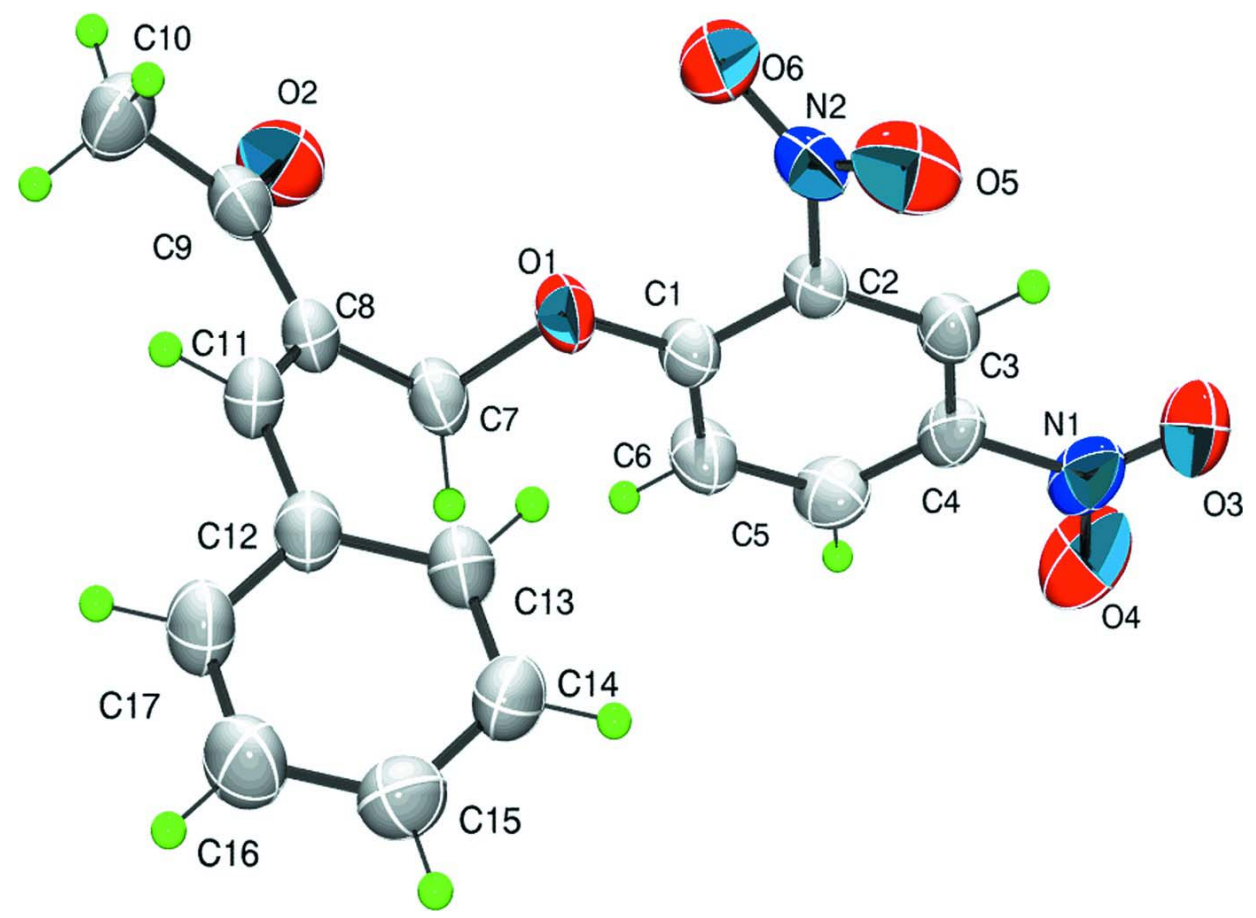

\section{Figure 1}

The molecular structure of the title showing the atom-labelling scheme and displacement ellipsoids at the $50 \%$ probability level.

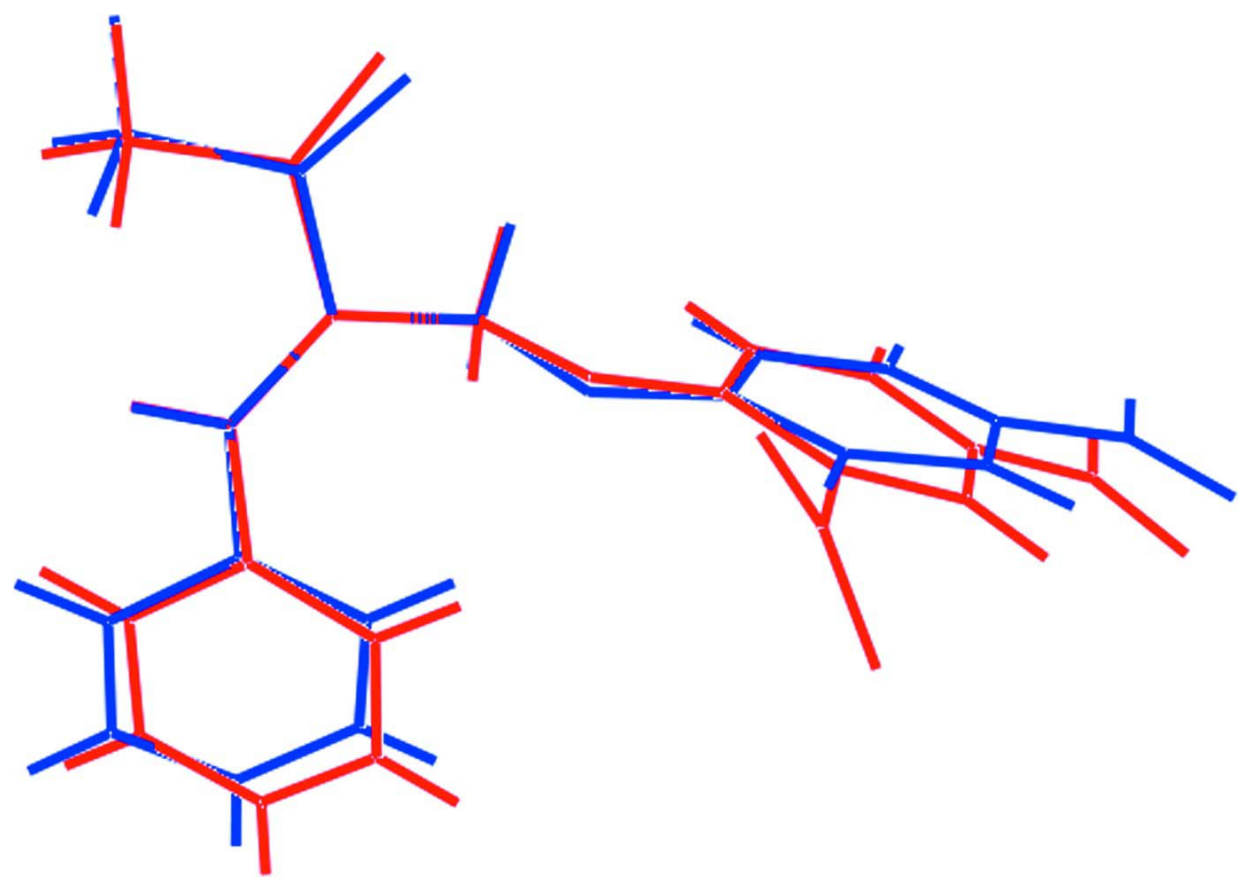

\section{Figure 2}

Overlay diagram of (I), red image, with inverted (II), blue image, drawn so that the $\mathrm{C}=C-C$ (phenyl) atoms are overlapped. 


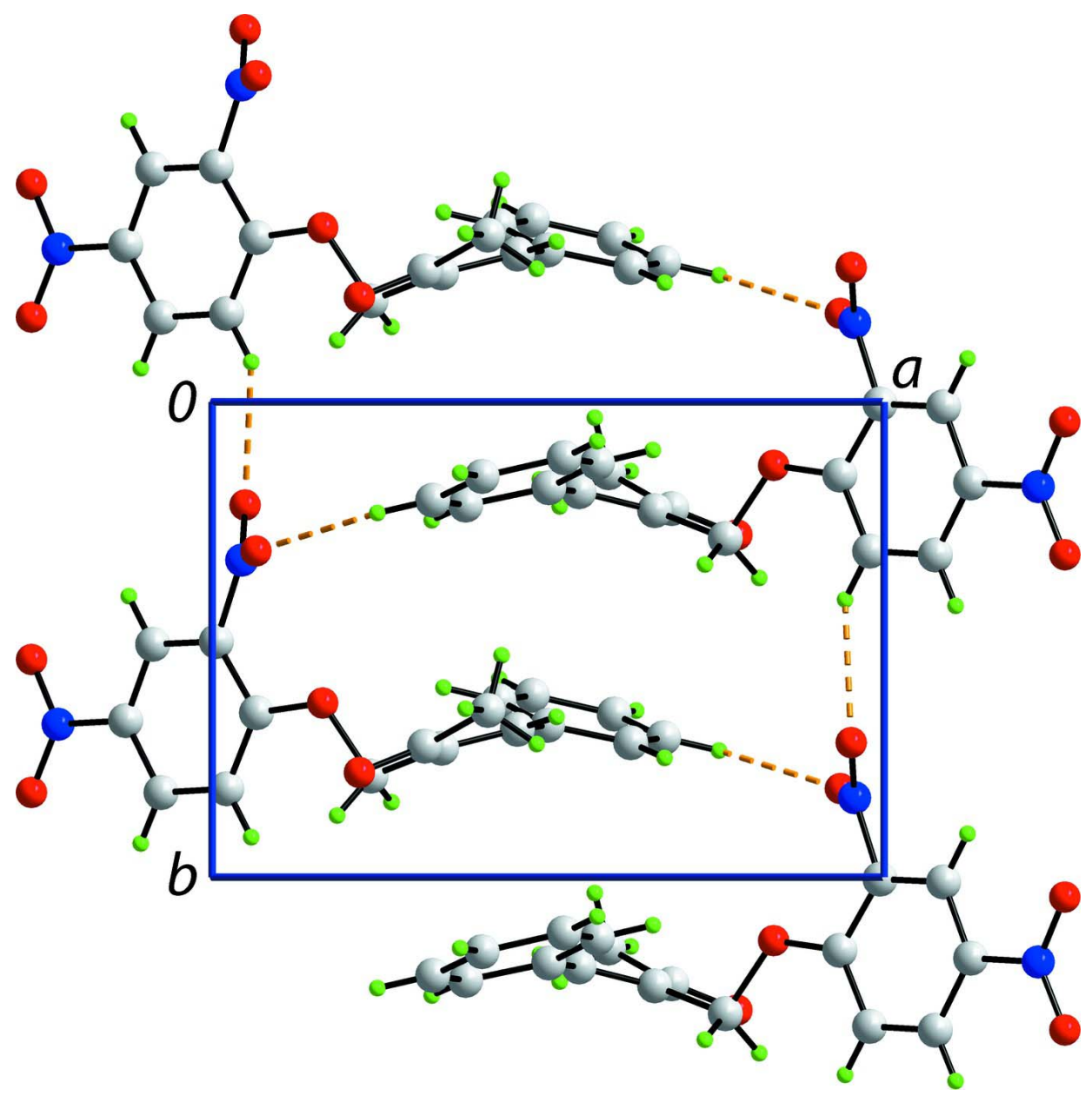

Figure 3

A view of helical supramolecular chain along $\left[\begin{array}{lll}0 & 1 & 0\end{array}\right]$. The $\mathrm{C}-\mathrm{H} \cdots \mathrm{O}$ contacts are shown as orange dashed lines. 


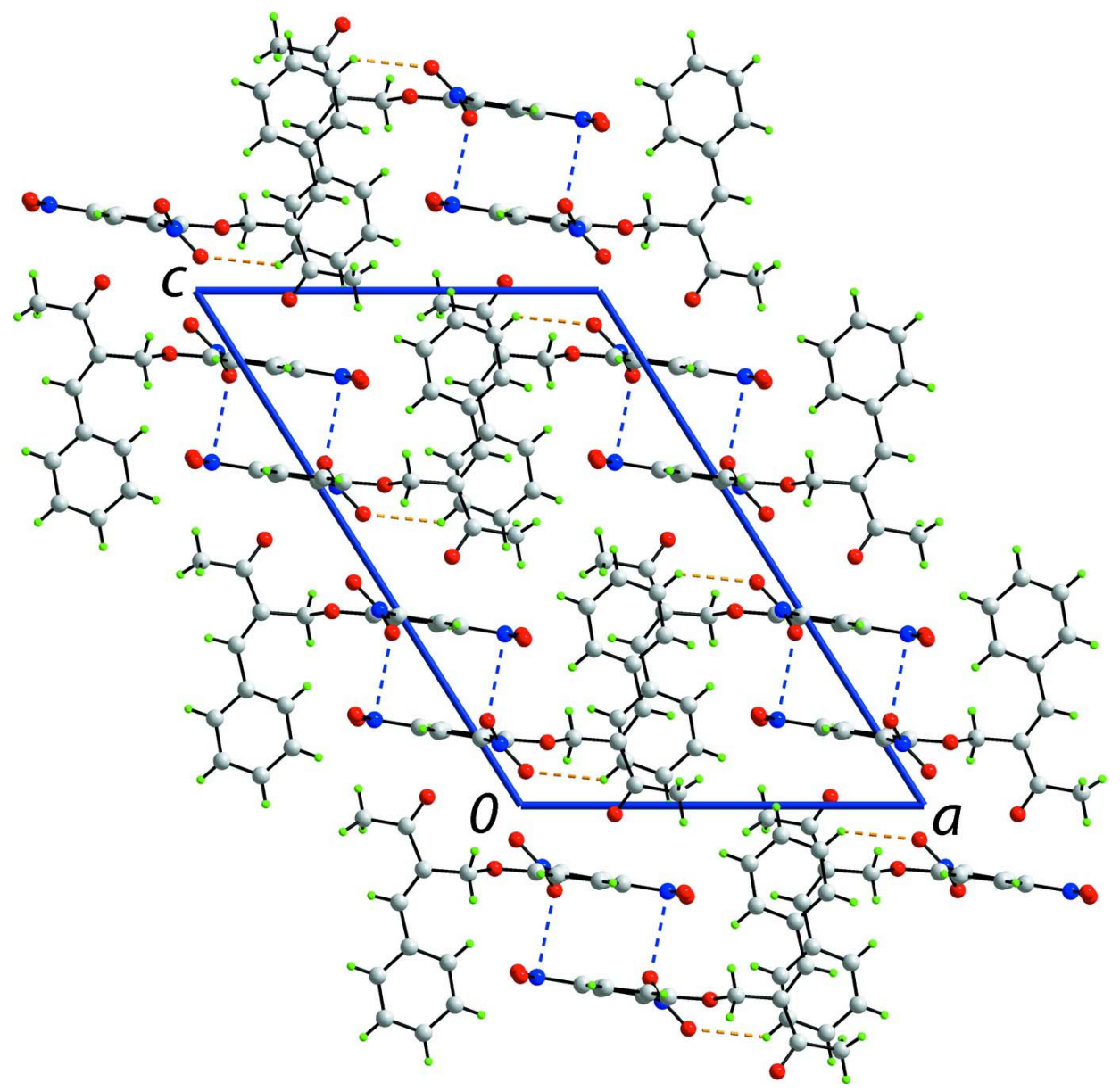

\section{Figure 4}

A view of unit-cell contents in projection down the $b$ axis. The $\mathrm{C}-\mathrm{H} \cdots \mathrm{O}$ and $\mathrm{N} \cdots \mathrm{O}$ contacts are shown as orange and blue dashed lines, respectively.

\section{(3E)-3-(2,4-Dinitrophenoxymethyl)-4-phenylbut-3-en-2-one}

\section{Crystal data}

$\mathrm{C}_{17} \mathrm{H}_{14} \mathrm{~N}_{2} \mathrm{O}_{6}$

$M_{r}=342.30$

Monoclinic, $P 2_{1} / c$

Hall symbol: $-\mathrm{P} 2 \mathrm{ybc}$

$a=12.8459$ (6) $\AA$

$b=7.6983(4) \AA$

$c=19.4283(8) \AA$

$\beta=122.254(2)^{\circ}$

$V=1624.82(14) \AA^{3}$

$Z=4$

\section{Data collection}

Bruker Kappa APEXII CCD diffractometer

Radiation source: fine-focus sealed tube Graphite monochromator $\omega$ and $\varphi$ scans
$F(000)=712$

$D_{\mathrm{x}}=1.399 \mathrm{Mg} \mathrm{m}^{-3}$

Mo $K \alpha$ radiation, $\lambda=0.71073 \AA$

Cell parameters from 6161 reflections

$\theta=2.9-25.3^{\circ}$

$\mu=0.11 \mathrm{~mm}^{-1}$

$T=290 \mathrm{~K}$

Irregular, colourless

$0.66 \times 0.45 \times 0.28 \mathrm{~mm}$

Absorption correction: multi-scan

(SADABS; Sheldrick, 1996)

$T_{\min }=0.699, T_{\max }=0.745$

10435 measured reflections

2957 independent reflections 
2630 reflections with $I>2 \sigma(I)$

$R_{\text {int }}=0.019$

$\theta_{\max }=25.3^{\circ}, \theta_{\min }=1.9^{\circ}$

\section{Refinement}

Refinement on $F^{2}$

Least-squares matrix: full

$R\left[F^{2}>2 \sigma\left(F^{2}\right)\right]=0.037$

$w R\left(F^{2}\right)=0.101$

$S=1.04$

2957 reflections

228 parameters

0 restraints

Primary atom site location: structure-invariant direct methods

Secondary atom site location: difference Fourier map

$$
\begin{aligned}
& h=-10 \rightarrow 15 \\
& k=-7 \rightarrow 9 \\
& l=-23 \rightarrow 19
\end{aligned}
$$

\section{Special details}

Geometry. All s.u.'s (except the s.u. in the dihedral angle between two 1.s. planes) are estimated using the full covariance matrix. The cell s.u.'s are taken into account individually in the estimation of s.u.'s in distances, angles and torsion angles; correlations between s.u.'s in cell parameters are only used when they are defined by crystal symmetry. An approximate (isotropic) treatment of cell s.u.'s is used for estimating s.u.'s involving 1.s. planes.

Refinement. Refinement of $F^{2}$ against ALL reflections. The weighted $R$-factor $w R$ and goodness of fit $S$ are based on $F^{2}$, conventional $R$-factors $R$ are based on $F$, with $F$ set to zero for negative $F^{2}$. The threshold expression of $F^{2}>2 \sigma\left(F^{2}\right)$ is used only for calculating $R$-factors(gt) etc. and is not relevant to the choice of reflections for refinement. $R$-factors based on $F^{2}$ are statistically about twice as large as those based on $F$, and $R$ - factors based on ALL data will be even larger.

Fractional atomic coordinates and isotropic or equivalent isotropic displacement parameters $\left(\AA^{2}\right)$

\begin{tabular}{lllll}
\hline & $x$ & $y$ & $z$ & $U_{\text {iso }} * / U_{\text {eq }}$ \\
\hline C1 & $0.93401(11)$ & $0.15407(17)$ & $0.37127(8)$ & $0.0384(3)$ \\
C2 & $0.99377(11)$ & $0.00425(16)$ & $0.36899(7)$ & $0.0364(3)$ \\
C3 & $1.08837(11)$ & $0.01022(18)$ & $0.35523(8)$ & $0.0409(3)$ \\
H3 & 1.1239 & -0.0912 & 0.3510 & $0.049^{*}$ \\
C4 & $1.12866(12)$ & $0.17061(19)$ & $0.34788(8)$ & $0.0436(3)$ \\
C5 & $1.07599(14)$ & $0.3221(2)$ & $0.35327(9)$ & $0.0509(4)$ \\
H5 & 1.1062 & 0.4292 & 0.3496 & $0.061^{*}$ \\
C6 & $0.97836(14)$ & $0.31403(18)$ & $0.36410(10)$ & $0.0494(4)$ \\
H6 & 0.9418 & 0.4161 & 0.3666 & $0.059^{*}$ \\
C7 & $0.76361(13)$ & $0.28733(18)$ & $0.36901(10)$ & $0.0463(3)$ \\
H7A & 0.7331 & 0.3422 & 0.3167 & $0.056^{*}$ \\
H7B & 0.8140 & 0.3705 & 0.4115 & $0.056^{*}$ \\
C8 & $0.65850(12)$ & $0.22873(19)$ & $0.37591(9)$ & $0.0437(3)$ \\
C9 & $0.68382(13)$ & $0.2244(2)$ & $0.46004(9)$ & $0.0503(4)$ \\
C10 & $0.58981(16)$ & $0.1491(3)$ & $0.47503(11)$ & $0.0656(5)$ \\
H10A & 0.6229 & 0.1458 & 0.5325 & $0.098^{*}$ \\
H10B & 0.5694 & 0.0334 & 0.4534 & $0.098^{*}$ \\
H10C & 0.5171 & 0.2199 & 0.4488 & $0.098^{*}$ \\
C11 & $0.54588(13)$ & $0.18897(19)$ & $0.31208(9)$ & $0.0464(3)$ \\
H11 & 0.4877 & 0.1628 & 0.3251 & $0.056^{*}$
\end{tabular}


supporting information

$\begin{array}{lllll}\text { C12 } & 0.50092(13) & 0.18058(18) & 0.22527(9) & 0.0447(3) \\ \text { C13 } & 0.57329(14) & 0.1286(2) & 0.19522(9) & 0.0502(4) \\ \text { H13 } & 0.6557 & 0.1012 & 0.2312 & 0.060^{*} \\ \text { C14 } & 0.52360(15) & 0.1174(2) & 0.11257(10) & 0.0568(4) \\ \text { H14 } & 0.5729 & 0.0830 & 0.0933 & 0.068^{*} \\ \text { C15 } & 0.40169(16) & 0.1568(2) & 0.05828(10) & 0.0596(4) \\ \text { H15 } & 0.3690 & 0.1496 & 0.0026 & 0.071^{*} \\ \text { C16 } & 0.32823(15) & 0.2069(2) & 0.08653(10) & 0.0613(4) \\ \text { H16 } & 0.2458 & 0.2337 & 0.0500 & 0.074^{*} \\ \text { C17 } & 0.37725(14) & 0.2172(2) & 0.16927(10) & 0.0539(4) \\ \text { H17 } & 0.3268 & 0.2492 & 0.1880 & 0.065^{*} \\ \text { N1 } & 1.22919(11) & 0.1784(2) & 0.33327(8) & 0.0572(4) \\ \text { N2 } & 0.95589(10) & -0.16711(15) & 0.38003(7) & 0.0443(3) \\ \text { O1 } & 0.83551(8) & 0.13359(12) & 0.37729(6) & 0.0459(3) \\ \text { O2 } & 0.78088(11) & 0.27977(19) & 0.51669(7) & 0.0716(4) \\ \text { O3 } & 1.26869(11) & 0.0420(2) & 0.32347(8) & 0.0767(4) \\ \text { O4 } & 1.26880(12) & 0.32170(19) & 0.33128(9) & 0.0825(4) \\ \text { O5 } & 0.95090(13) & -0.28306(15) & 0.33557(8) & 0.0716(4) \\ \text { O6 } & 0.93475(11) & -0.18635(15) & 0.43354(8) & 0.0673(4)\end{array}$

Atomic displacement parameters $\left(\AA^{2}\right)$

\begin{tabular}{|c|c|c|c|c|c|c|}
\hline & $U^{11}$ & $U^{22}$ & $U^{33}$ & $U^{12}$ & $U^{13}$ & $U^{23}$ \\
\hline $\mathrm{C} 1$ & $0.0332(6)$ & $0.0384(7)$ & $0.0442(7)$ & $-0.0002(5)$ & $0.0209(6)$ & $0.0030(5)$ \\
\hline $\mathrm{C} 2$ & $0.0316(6)$ & $0.0352(7)$ & $0.0391(6)$ & $-0.0014(5)$ & $0.0167(5)$ & $0.0027(5)$ \\
\hline $\mathrm{C} 3$ & $0.0341(6)$ & $0.0469(8)$ & $0.0400(7)$ & $0.0039(6)$ & $0.0187(6)$ & $0.0018(6)$ \\
\hline $\mathrm{C} 4$ & $0.0338(6)$ & $0.0564(9)$ & $0.0412(7)$ & $-0.0039(6)$ & $0.0204(6)$ & $0.0042(6)$ \\
\hline $\mathrm{C} 5$ & $0.0478(8)$ & $0.0461(8)$ & $0.0605(9)$ & $-0.0090(6)$ & $0.0301(7)$ & $0.0067(7)$ \\
\hline C6 & $0.0496(8)$ & $0.0357(7)$ & $0.0681(10)$ & $0.0009(6)$ & $0.0349(8)$ & $0.0041(7)$ \\
\hline $\mathrm{C} 7$ & $0.0426(7)$ & $0.0407(8)$ & $0.0599(9)$ & $0.0080(6)$ & $0.0302(7)$ & $0.0032(6)$ \\
\hline C8 & $0.0405(7)$ & $0.0443(8)$ & $0.0518(8)$ & $0.0104(6)$ & $0.0284(6)$ & $0.0041(6)$ \\
\hline C9 & $0.0443(8)$ & $0.0550(9)$ & $0.0509(8)$ & $0.0158(7)$ & $0.0248(7)$ & $0.0081(7)$ \\
\hline $\mathrm{C} 10$ & $0.0647(10)$ & $0.0875(13)$ & $0.0582(10)$ & $0.0071(9)$ & $0.0419(9)$ & $0.0055(9)$ \\
\hline $\mathrm{C} 11$ & $0.0418(7)$ & $0.0524(8)$ & $0.0542(8)$ & $0.0061(6)$ & $0.0318(7)$ & $0.0013(6)$ \\
\hline $\mathrm{C} 12$ & $0.0436(7)$ & $0.0443(8)$ & $0.0517(8)$ & $0.0024(6)$ & $0.0291(7)$ & $0.0003(6)$ \\
\hline C13 & $0.0470(8)$ & $0.0546(9)$ & $0.0541(8)$ & $0.0103(7)$ & $0.0304(7)$ & $0.0040(7)$ \\
\hline $\mathrm{C} 14$ & $0.0668(10)$ & $0.0592(10)$ & $0.0597(9)$ & $0.0106(8)$ & $0.0440(8)$ & $0.0052(8)$ \\
\hline $\mathrm{C} 15$ & $0.0666(10)$ & $0.0640(11)$ & $0.0492(9)$ & $0.0053(8)$ & $0.0317(8)$ & $0.0051(7)$ \\
\hline $\mathrm{C} 16$ & $0.0463(8)$ & $0.0755(12)$ & $0.0553(9)$ & $0.0049(8)$ & $0.0226(7)$ & $0.0018(8)$ \\
\hline $\mathrm{C} 17$ & $0.0418(7)$ & $0.0650(10)$ & $0.0591(9)$ & $0.0018(7)$ & $0.0298(7)$ & $-0.0040(7)$ \\
\hline N1 & $0.0398(7)$ & $0.0847(11)$ & $0.0497(7)$ & $-0.0076(7)$ & $0.0256(6)$ & $0.0055(7)$ \\
\hline N2 & $0.0342(6)$ & $0.0364(6)$ & $0.0536(7)$ & $0.0010(5)$ & $0.0177(5)$ & $0.0058(5)$ \\
\hline O1 & $0.0380(5)$ & $0.0394(5)$ & $0.0685(6)$ & 0.0048 (4) & $0.0339(5)$ & $0.0072(4)$ \\
\hline $\mathrm{O} 2$ & $0.0542(7)$ & $0.0928(10)$ & $0.0513(7)$ & $0.0044(6)$ & $0.0170(6)$ & $0.0071(6)$ \\
\hline $\mathrm{O} 3$ & $0.0611(7)$ & $0.0984(10)$ & $0.0919(9)$ & $0.0169(7)$ & $0.0550(7)$ & $0.0151(8)$ \\
\hline $\mathrm{O} 4$ & $0.0699(8)$ & $0.0988(11)$ & $0.0971(10)$ & $-0.0359(7)$ & $0.0567(8)$ & $-0.0084(8)$ \\
\hline O5 & $0.0896(9)$ & $0.0395(6)$ & $0.0671(8)$ & $-0.0070(6)$ & $0.0294(7)$ & $-0.0080(5)$ \\
\hline O6 & $0.0747(8)$ & $0.0542(7)$ & $0.0984(9)$ & $0.0033(6)$ & $0.0632(8)$ & $0.0199(6)$ \\
\hline
\end{tabular}


Geometric parameters $\left(\AA,{ }^{\circ}\right)$

\begin{tabular}{|c|c|c|c|}
\hline $\mathrm{C} 1-\mathrm{O} 1$ & $1.3405(15)$ & $\mathrm{C} 10-\mathrm{H} 10 \mathrm{~A}$ & 0.9600 \\
\hline $\mathrm{C} 1-\mathrm{C} 6$ & $1.3947(18)$ & $\mathrm{C} 10-\mathrm{H} 10 \mathrm{~B}$ & 0.9600 \\
\hline $\mathrm{C} 1-\mathrm{C} 2$ & $1.3993(18)$ & $\mathrm{C} 10-\mathrm{H} 10 \mathrm{C}$ & 0.9600 \\
\hline $\mathrm{C} 2-\mathrm{C} 3$ & $1.3773(17)$ & $\mathrm{C} 11-\mathrm{C} 12$ & $1.464(2)$ \\
\hline $\mathrm{C} 2-\mathrm{N} 2$ & $1.4610(16)$ & $\mathrm{C} 11-\mathrm{H} 11$ & 0.9300 \\
\hline $\mathrm{C} 3-\mathrm{C} 4$ & $1.375(2)$ & $\mathrm{C} 12-\mathrm{C} 17$ & $1.394(2)$ \\
\hline $\mathrm{C} 3-\mathrm{H} 3$ & 0.9300 & $\mathrm{C} 12-\mathrm{C} 13$ & 1.3945 (19) \\
\hline $\mathrm{C} 4-\mathrm{C} 5$ & $1.380(2)$ & $\mathrm{C} 13-\mathrm{C} 14$ & $1.378(2)$ \\
\hline $\mathrm{C} 4-\mathrm{N} 1$ & $1.4638(17)$ & $\mathrm{C} 13-\mathrm{H} 13$ & 0.9300 \\
\hline $\mathrm{C} 5-\mathrm{C} 6$ & $1.379(2)$ & $\mathrm{C} 14-\mathrm{C} 15$ & $1.376(2)$ \\
\hline $\mathrm{C} 5-\mathrm{H} 5$ & 0.9300 & $\mathrm{C} 14-\mathrm{H} 14$ & 0.9300 \\
\hline $\mathrm{C} 6-\mathrm{H} 6$ & 0.9300 & $\mathrm{C} 15-\mathrm{C} 16$ & $1.376(2)$ \\
\hline $\mathrm{C} 7-\mathrm{O} 1$ & $1.4565(16)$ & $\mathrm{C} 15-\mathrm{H} 15$ & 0.9300 \\
\hline $\mathrm{C} 7-\mathrm{C} 8$ & $1.4959(19)$ & $\mathrm{C} 16-\mathrm{C} 17$ & $1.381(2)$ \\
\hline C7-H7A & 0.9700 & $\mathrm{C} 16-\mathrm{H} 16$ & 0.9300 \\
\hline C7-H7B & 0.9700 & $\mathrm{C} 17-\mathrm{H} 17$ & 0.9300 \\
\hline $\mathrm{C} 8-\mathrm{C} 11$ & $1.345(2)$ & $\mathrm{N} 1-\mathrm{O} 4$ & $1.2242(18)$ \\
\hline $\mathrm{C} 8-\mathrm{C} 9$ & $1.487(2)$ & $\mathrm{N} 1-\mathrm{O} 3$ & $1.2242(19)$ \\
\hline $\mathrm{C} 9-\mathrm{O} 2$ & $1.2176(19)$ & $\mathrm{N} 2-\mathrm{O} 6$ & $1.2156(16)$ \\
\hline $\mathrm{C} 9-\mathrm{C} 10$ & $1.502(2)$ & $\mathrm{N} 2-\mathrm{O} 5$ & $1.2198(16)$ \\
\hline $\mathrm{O} 1-\mathrm{C} 1-\mathrm{C} 6$ & $124.58(12)$ & $\mathrm{H} 10 \mathrm{~A}-\mathrm{C} 10-\mathrm{H} 10 \mathrm{~B}$ & 109.5 \\
\hline $\mathrm{O} 1-\mathrm{C} 1-\mathrm{C} 2$ & $117.72(11)$ & $\mathrm{C} 9-\mathrm{C} 10-\mathrm{H} 10 \mathrm{C}$ & 109.5 \\
\hline $\mathrm{C} 6-\mathrm{C} 1-\mathrm{C} 2$ & $117.66(12)$ & $\mathrm{H} 10 \mathrm{~A}-\mathrm{C} 10-\mathrm{H} 10 \mathrm{C}$ & 109.5 \\
\hline $\mathrm{C} 3-\mathrm{C} 2-\mathrm{C} 1$ & $122.31(12)$ & $\mathrm{H} 10 \mathrm{~B}-\mathrm{C} 10-\mathrm{H} 10 \mathrm{C}$ & 109.5 \\
\hline $\mathrm{C} 3-\mathrm{C} 2-\mathrm{N} 2$ & $117.14(11)$ & $\mathrm{C} 8-\mathrm{C} 11-\mathrm{C} 12$ & $129.87(13)$ \\
\hline $\mathrm{C} 1-\mathrm{C} 2-\mathrm{N} 2$ & $120.55(11)$ & $\mathrm{C} 8-\mathrm{C} 11-\mathrm{H} 11$ & 115.1 \\
\hline $\mathrm{C} 4-\mathrm{C} 3-\mathrm{C} 2$ & $118.04(12)$ & $\mathrm{C} 12-\mathrm{C} 11-\mathrm{H} 11$ & 115.1 \\
\hline $\mathrm{C} 4-\mathrm{C} 3-\mathrm{H} 3$ & 121.0 & $\mathrm{C} 17-\mathrm{C} 12-\mathrm{C} 13$ & $117.91(13)$ \\
\hline $\mathrm{C} 2-\mathrm{C} 3-\mathrm{H} 3$ & 121.0 & $\mathrm{C} 17-\mathrm{C} 12-\mathrm{C} 11$ & $118.36(12)$ \\
\hline $\mathrm{C} 3-\mathrm{C} 4-\mathrm{C} 5$ & $121.58(12)$ & $\mathrm{C} 13-\mathrm{C} 12-\mathrm{C} 11$ & $123.63(13)$ \\
\hline $\mathrm{C} 3-\mathrm{C} 4-\mathrm{N} 1$ & $118.47(13)$ & $\mathrm{C} 14-\mathrm{C} 13-\mathrm{C} 12$ & $120.53(14)$ \\
\hline $\mathrm{C} 5-\mathrm{C} 4-\mathrm{N} 1$ & $119.95(13)$ & $\mathrm{C} 14-\mathrm{C} 13-\mathrm{H} 13$ & 119.7 \\
\hline $\mathrm{C} 6-\mathrm{C} 5-\mathrm{C} 4$ & $119.75(13)$ & $\mathrm{C} 12-\mathrm{C} 13-\mathrm{H} 13$ & 119.7 \\
\hline $\mathrm{C} 6-\mathrm{C} 5-\mathrm{H} 5$ & 120.1 & $\mathrm{C} 15-\mathrm{C} 14-\mathrm{C} 13$ & $120.61(14)$ \\
\hline $\mathrm{C} 4-\mathrm{C} 5-\mathrm{H} 5$ & 120.1 & $\mathrm{C} 15-\mathrm{C} 14-\mathrm{H} 14$ & 119.7 \\
\hline $\mathrm{C} 5-\mathrm{C} 6-\mathrm{C} 1$ & $120.54(13)$ & $\mathrm{C} 13-\mathrm{C} 14-\mathrm{H} 14$ & 119.7 \\
\hline $\mathrm{C} 5-\mathrm{C} 6-\mathrm{H} 6$ & 119.7 & $\mathrm{C} 16-\mathrm{C} 15-\mathrm{C} 14$ & $119.88(15)$ \\
\hline $\mathrm{C} 1-\mathrm{C} 6-\mathrm{H} 6$ & 119.7 & $\mathrm{C} 16-\mathrm{C} 15-\mathrm{H} 15$ & 120.1 \\
\hline $\mathrm{O} 1-\mathrm{C} 7-\mathrm{C} 8$ & $107.14(11)$ & $\mathrm{C} 14-\mathrm{C} 15-\mathrm{H} 15$ & 120.1 \\
\hline $\mathrm{O} 1-\mathrm{C} 7-\mathrm{H} 7 \mathrm{~A}$ & 110.3 & $\mathrm{C} 15-\mathrm{C} 16-\mathrm{C} 17$ & $119.79(15)$ \\
\hline $\mathrm{C} 8-\mathrm{C} 7-\mathrm{H} 7 \mathrm{~A}$ & 110.3 & $\mathrm{C} 15-\mathrm{C} 16-\mathrm{H} 16$ & 120.1 \\
\hline $\mathrm{O} 1-\mathrm{C} 7-\mathrm{H} 7 \mathrm{~B}$ & 110.3 & $\mathrm{C} 17-\mathrm{C} 16-\mathrm{H} 16$ & 120.1 \\
\hline $\mathrm{C} 8-\mathrm{C} 7-\mathrm{H} 7 \mathrm{~B}$ & 110.3 & $\mathrm{C} 16-\mathrm{C} 17-\mathrm{C} 12$ & $121.26(14)$ \\
\hline $\mathrm{H} 7 \mathrm{~A}-\mathrm{C} 7-\mathrm{H} 7 \mathrm{~B}$ & 108.5 & $\mathrm{C} 16-\mathrm{C} 17-\mathrm{H} 17$ & 119.4 \\
\hline $\mathrm{C} 11-\mathrm{C} 8-\mathrm{C} 9$ & $120.33(12)$ & $\mathrm{C} 12-\mathrm{C} 17-\mathrm{H} 17$ & 119.4 \\
\hline
\end{tabular}




\begin{tabular}{|c|c|c|c|}
\hline $\mathrm{C} 11-\mathrm{C} 8-\mathrm{C} 7$ & $124.35(13)$ & $\mathrm{O} 4-\mathrm{N} 1-\mathrm{O} 3$ & $123.64(13)$ \\
\hline $\mathrm{C} 9-\mathrm{C} 8-\mathrm{C} 7$ & $115.26(13)$ & $\mathrm{O} 4-\mathrm{N} 1-\mathrm{C} 4$ & $117.88(15)$ \\
\hline $\mathrm{O} 2-\mathrm{C} 9-\mathrm{C} 8$ & $120.24(14)$ & $\mathrm{O} 3-\mathrm{N} 1-\mathrm{C} 4$ & $118.49(13)$ \\
\hline $\mathrm{O} 2-\mathrm{C} 9-\mathrm{C} 10$ & $120.09(14)$ & $\mathrm{O} 6-\mathrm{N} 2-\mathrm{O} 5$ & $124.41(13)$ \\
\hline $\mathrm{C} 8-\mathrm{C} 9-\mathrm{C} 10$ & $119.67(14)$ & $\mathrm{O} 6-\mathrm{N} 2-\mathrm{C} 2$ & $118.65(12)$ \\
\hline $\mathrm{C} 9-\mathrm{C} 10-\mathrm{H} 10 \mathrm{~A}$ & 109.5 & $\mathrm{O} 5-\mathrm{N} 2-\mathrm{C} 2$ & $116.90(12)$ \\
\hline $\mathrm{C} 9-\mathrm{C} 10-\mathrm{H} 10 \mathrm{~B}$ & 109.5 & $\mathrm{C} 1-\mathrm{O} 1-\mathrm{C} 7$ & $117.86(10)$ \\
\hline $\mathrm{O} 1-\mathrm{C} 1-\mathrm{C} 2-\mathrm{C} 3$ & $173.75(11)$ & $\mathrm{C} 8-\mathrm{C} 11-\mathrm{C} 12-\mathrm{C} 17$ & $-150.23(16)$ \\
\hline $\mathrm{C} 6-\mathrm{C} 1-\mathrm{C} 2-\mathrm{C} 3$ & $-3.9(2)$ & $\mathrm{C} 8-\mathrm{C} 11-\mathrm{C} 12-\mathrm{C} 13$ & $33.5(2)$ \\
\hline $\mathrm{O} 1-\mathrm{C} 1-\mathrm{C} 2-\mathrm{N} 2$ & $-5.24(18)$ & $\mathrm{C} 17-\mathrm{C} 12-\mathrm{C} 13-\mathrm{C} 14$ & $1.2(2)$ \\
\hline $\mathrm{C} 6-\mathrm{C} 1-\mathrm{C} 2-\mathrm{N} 2$ & $177.08(12)$ & $\mathrm{C} 11-\mathrm{C} 12-\mathrm{C} 13-\mathrm{C} 14$ & $177.53(15)$ \\
\hline $\mathrm{C} 1-\mathrm{C} 2-\mathrm{C} 3-\mathrm{C} 4$ & $3.50(19)$ & $\mathrm{C} 12-\mathrm{C} 13-\mathrm{C} 14-\mathrm{C} 15$ & $-0.2(3)$ \\
\hline $\mathrm{N} 2-\mathrm{C} 2-\mathrm{C} 3-\mathrm{C} 4$ & $-177.48(11)$ & $\mathrm{C} 13-\mathrm{C} 14-\mathrm{C} 15-\mathrm{C} 16$ & $-0.4(3)$ \\
\hline $\mathrm{C} 2-\mathrm{C} 3-\mathrm{C} 4-\mathrm{C} 5$ & $-0.6(2)$ & $\mathrm{C} 14-\mathrm{C} 15-\mathrm{C} 16-\mathrm{C} 17$ & $-0.1(3)$ \\
\hline $\mathrm{C} 2-\mathrm{C} 3-\mathrm{C} 4-\mathrm{N} 1$ & $179.98(11)$ & $\mathrm{C} 15-\mathrm{C} 16-\mathrm{C} 17-\mathrm{C} 12$ & $1.1(3)$ \\
\hline $\mathrm{C} 3-\mathrm{C} 4-\mathrm{C} 5-\mathrm{C} 6$ & $-1.8(2)$ & $\mathrm{C} 13-\mathrm{C} 12-\mathrm{C} 17-\mathrm{C} 16$ & $-1.7(2)$ \\
\hline $\mathrm{N} 1-\mathrm{C} 4-\mathrm{C} 5-\mathrm{C} 6$ & $177.66(13)$ & $\mathrm{C} 11-\mathrm{C} 12-\mathrm{C} 17-\mathrm{C} 16$ & $-178.17(15)$ \\
\hline $\mathrm{C} 4-\mathrm{C} 5-\mathrm{C} 6-\mathrm{C} 1$ & $1.3(2)$ & $\mathrm{C} 3-\mathrm{C} 4-\mathrm{N} 1-\mathrm{O} 4$ & $-176.08(13)$ \\
\hline $\mathrm{O} 1-\mathrm{C} 1-\mathrm{C} 6-\mathrm{C} 5$ & $-176.05(13)$ & $\mathrm{C} 5-\mathrm{C} 4-\mathrm{N} 1-\mathrm{O} 4$ & $4.5(2)$ \\
\hline $\mathrm{C} 2-\mathrm{C} 1-\mathrm{C} 6-\mathrm{C} 5$ & $1.5(2)$ & $\mathrm{C} 3-\mathrm{C} 4-\mathrm{N} 1-\mathrm{O} 3$ & $4.2(2)$ \\
\hline $\mathrm{O} 1-\mathrm{C} 7-\mathrm{C} 8-\mathrm{C} 11$ & $-94.16(16)$ & $\mathrm{C} 5-\mathrm{C} 4-\mathrm{N} 1-\mathrm{O} 3$ & $-175.29(14)$ \\
\hline $\mathrm{O} 1-\mathrm{C} 7-\mathrm{C} 8-\mathrm{C} 9$ & $88.75(14)$ & $\mathrm{C} 3-\mathrm{C} 2-\mathrm{N} 2-\mathrm{O} 6$ & $137.34(13)$ \\
\hline $\mathrm{C} 11-\mathrm{C} 8-\mathrm{C} 9-\mathrm{O} 2$ & $-171.36(15)$ & $\mathrm{C} 1-\mathrm{C} 2-\mathrm{N} 2-\mathrm{O} 6$ & $-43.62(17)$ \\
\hline $\mathrm{C} 7-\mathrm{C} 8-\mathrm{C} 9-\mathrm{O} 2$ & $5.9(2)$ & $\mathrm{C} 3-\mathrm{C} 2-\mathrm{N} 2-\mathrm{O} 5$ & $-40.76(17)$ \\
\hline $\mathrm{C} 11-\mathrm{C} 8-\mathrm{C} 9-\mathrm{C} 10$ & $9.5(2)$ & $\mathrm{C} 1-\mathrm{C} 2-\mathrm{N} 2-\mathrm{O} 5$ & $138.28(13)$ \\
\hline $\mathrm{C} 7-\mathrm{C} 8-\mathrm{C} 9-\mathrm{C} 10$ & $-173.26(13)$ & $\mathrm{C} 6-\mathrm{C} 1-\mathrm{O} 1-\mathrm{C} 7$ & $5.4(2)$ \\
\hline $\mathrm{C} 9-\mathrm{C} 8-\mathrm{C} 11-\mathrm{C} 12$ & $-178.44(14)$ & $\mathrm{C} 2-\mathrm{C} 1-\mathrm{O} 1-\mathrm{C} 7$ & $-172.16(12)$ \\
\hline $\mathrm{C} 7-\mathrm{C} 8-\mathrm{C} 11-\mathrm{C} 12$ & $4.6(2)$ & $\mathrm{C} 8-\mathrm{C} 7-\mathrm{O} 1-\mathrm{C} 1$ & $178.57(11)$ \\
\hline
\end{tabular}

Hydrogen-bond geometry $\left(\AA,{ }^{\circ}\right)$

\begin{tabular}{lllll}
\hline$D-\mathrm{H} \cdots A$ & $D-\mathrm{H}$ & $\mathrm{H} \cdots A$ & $D \cdots A$ & $D-\mathrm{H} \cdots A$ \\
\hline $\mathrm{C} 6-\mathrm{H} 6 \cdots \mathrm{O}^{\mathrm{i}}$ & 0.93 & 2.41 & $3.1375(18)$ & 135 \\
$\mathrm{C} 16-\mathrm{H} 16 \cdots \mathrm{O}^{\mathrm{ii}}$ & 0.93 & 2.58 & $3.292(3)$ & 134 \\
\hline
\end{tabular}

Symmetry codes: (i) $x, y+1, z$; (ii) $-x+1, y+1 / 2,-z+1 / 2$. 